\title{
THE ADDED VALUE OF INTEGRATE-HTA GUIDANCE IN THE WORK PROCESSES OF HEALTH TECHNOLOGY ASSESSMENT AGENCIES
}

Gert Jan van der Wilt

Donders Institute for Brain, Cognition, and Behaviour

gertjan.vanderwilt@radboudumc.nl

\author{
Wietske Kievit \\ Radboud Institute for Health Sciences \\ Wija Oortwiin \\ Ecorys Netherlands
}

A central idea underlying the INTEGRATE-HTA project is that many of the interventions that are being used in health care are quite complex. By this, we mean that the relation between the delivery of the intervention on the one hand, and the onset of (desired and undesired) changes may be less straightforward than hoped for. There may be all sorts of reasons for this, varying from a lack of resources, lack of skills, perverse incentives, organizational problems, etc. Not identifying such factors and their potential impact may seriously compromise the policy relevance of a health technology assessment (HTA) (1). However, current approaches and methods in HTA do not seem to be adequately geared to deal with this complexity.

The INTEGRATE-HTA project has developed and tested several tools that can assist HTA researchers in exploring complexity and its possible implications for health interventions. A scoping exercise, conducted at the start of an HTA and involving the various relevant stakeholders, may help to identify potential sources of complexity and provide guidance to areas for further inquiry. Developing logic models may help to synthesize the various understandings of the relevant issues involved, graphically representing how interventions are thought to result in possible outcomes, spelling out what contextual factors are thought to moderate these processes, in what way, to what extent, and under what conditions. Any indications for interdependencies may then be the subject of further inquiry, and findings can be shared with organizations commissioning HTA. The question is, however: could such an approach be incorporated in the workflow of HTA agencies, and would it fit within their remit?

A fact of life is that HTA agencies are faced with mounting pressure to deliver within increasingly tight time schedules, and commissioning organizations may not have the patience to wait for the results of more extensive analyses. Stakeholder involvement may enhance the policy relevance of an HTA and the legitimacy of subsequent policy decisions, but it will almost certainly increase the lead time of an HTA. In other words, does the INTEGRATE-HTA guidance take sufficient account of the complexity of real-world HTA? Or does it reflect an ideal that is of academic interest, but impossible to implement in practice?

To explore those issues, we organized an INAHTA (International Network of Agencies for Health Technology Assessment) webinar on INTEGRATE-HTA, in which colleagues from the Canadian Agency for Drugs and Health Technologies shared their experiences with using the guidance developed in the INTEGRATE-HTA project. See for more information, the article presented in this theme issue (2). Furthermore, a delegation of the INTEGRATE-HTA team (the authors of this contribution) paid a visit to one of the national HTA agencies in Europe, the Belgian Health Care Knowledge Centre (KCE) and had an interview with two members of the staff. The KCE participates in EUnetHTA, being the co-lead partner of quality management, scientific guidance and tools in this consortium. As such, we believe that their perspective on the INTEGRATEHTA project may be reflective of HTA agencies more generally, particularly in Europe. Below, we present a summary of the interview to indicate if and how the guidance of INTEGRATEHTA could be of relevance and adopted in their work process.

\section{We: Could you briefly describe your organization?}

KCE: The KCE consists of a team of ca. sixty people, among which more than forty researchers. Its mission is to advise, on the basis of scientific analyses, policy makers on decisions relating to health care and health insurance. Its role is to identify the best possible solutions for prespecified health care problems, taking into account accessibility, quality, a growing demand and budgetary constraints. Furthermore, KCE supports 
care providers by developing clinical guidelines, gearing these toward the evolving body of scientific knowledge and publishing on methodologies that serve as a guide for other health care researchers (for further details, see kce.fgov.be).

\section{We: Do you recognize the rationale of the INTEGRATE-HTA project, the complexity of health interventions, and its implications for HTA?}

KCE: Yes, we do. For example, KCE recently launched a report on the evaluation and management of low back pain and radicular pain. We concluded that there are important opportunities for improving the quality and efficiency of care for these patients. At the same time, we do recognize some key barriers. For instance, health care professionals will need more training in stratifying patients into different risk categories. Also, patients' expectations may thwart necessary changes. They may demand imaging, and it may be very difficult for healthcare professionals to resist such demands. A paradigm shift from a biomedical to a biopsychosocial approach is needed, and this will take time. In addition, multidisciplinary programs are needed, and patients should be encouraged to continue daily activities, including work. Hence, although the evidence concerning the effectiveness of specific health care programs seems to be fairly straightforward, allowing for the development of guidelines, implementation of such guidelines will be challenging.

\section{We: Do you think the INTEGRATE-HTA could be useful in meeting those challenges?}

KCE: We do acknowledge the importance of stakeholder involvement in HTA. This seems to be one of the elements of the INTEGRATE-HTA approach, most prominently in its guidance on scoping. We also are investing a lot in stakeholder involvement, and our general impression is that it may enhance support for and uptake of guidelines. However, there is always a trade-off, and an increased expenditure of time and resources needs to be balanced with anticipated benefits. In addition, the guidance on building logic models could be useful.

It is akin to the infographics that we increasingly use to summarize the main findings of our analyses. It allows you to graphically represent processes, work flows, ideas about mechanisms, etc. We used them in a report on multi-criteria decision analysis (MCDA), to summarize current processes for early temporary reimbursement, and to summarize the MCDA process itself. For us, conceptually, the most appealing elements of the INTEGRATE-HTA project are complexity, multidimensionality, and context (A similar point is made by Bond and Weeks, who have been using the INTEGRATE-HTA guidance at the Canadian Agency for Drugs and Technologies in Health (CADTH) (2).

\section{We: What do you consider the major challenges for HTA in the near future, and how might INTEGRATE-HTA help to meet those challenges?}

$\mathrm{KCE}$ : A key challenge is to do more work in less time. We do not see how INTEGRATE-HTA helps to meet that challenge. Secondly, we are seeing a shift from traditional HTA toward what we would call Health Services Research (HSR). The difference being that the research question shifts from "What are the relevant aspects of this health technology that need to be taken into account to inform a decision?" (= HTA) to "How will this health technology affect the system and the health prospects of the population?" (= HSR). We think that this is an important, indeed desirable, change, resulting in outcomes that are more useful and relevant to policymakers.

In a way, we have to return to the original concept of HTA, as formulated by for instance David Banta, emphasizing that HTA is a type of policy research, identifying and evaluating different policy options (3). However, this is clearly a much more complex task, also setting different demands on the researchers. It would be important to redirect HTA educational programs to better prepare a future generation of HTA researchers to face those challenges. we think that INTEGRATE-HTA definitely has something to offer here, both in terms of methodology, and in terms of thinking about health and healthcare (conceptually).

Finally, we have to admit that we are still struggling with ethics. The mission of KCE is to produce useful, intelligent and completely state-of-the-art analyses of complex health(care) problems, to indicate potential solutions, and to provide an overview of the relative advantages and disadvantages that are associated with those solutions. We cannot, nor desire, make those decisions ourselves. Can we, at KCE, inform policy makers about the ethical implications of various policy options, in a way that is nondirective? So far, we have been experimenting with the accountability for reasonableness approach, developed by Norman Daniels (4). We found this useful, but do see its limitations. We would be interested to see whether the INTEGRATE-HTA has something to offer here that could help us in navigating between "being insufficient" and "overreaching," as Norman Daniels put it (5).

\section{REFERENCES}

1. Brereton $\mathrm{L}$, et al, this issue.

2. Bond K, Weeks L. Using the INTEHRATE-HTA Guidance: Experience from CADTH. This volume.

3. Banta HD, Luce BR. Health care technology and its assessment. An international perspective. Oxford: Oxford University Press; 1993:61.

4. Daniels N, Sabin J, Accountability for Reasonableness: an update. BMJ. 2008;337:a1850.

5. Daniels N, van der Wil GJ, Health Technology Assessment, deliberative process, and ethically contested issues. Int J Technol Assess Health Care. 2016;32:10-15. (See also the contribution of Bond and Weeks in this volume, reporting on their experience with using the INTEGRATEHTA guidance on addressing ethical issues in the context of HTA) 